\title{
Virulence of metalloproteases produced by Vibrio species on Pacific oyster Crassostrea gigas larvae
}

\author{
Hiroaki Hasegawa, Dima N. Gharaibeh, Erin J. Lind, Claudia C. Häse* \\ Department of Biomedical Sciences, Oregon State University, Corvallis, Oregon 97331
}

\begin{abstract}
Vibrio tubiashii, a pathogen of shellfish larvae and juveniles, produces several extracellular products. Here, we document that culture supernatants of several marine Vibrio species showed toxicity to oyster larvae. Treatment of these supernatants with EDTA not only severely diminished proteolytic activities, but also dramatically reduced toxicity to the larvae. Culture supernatants of metalloprotease-deficient mutants of $V$. tubiashii, $V$. cholerae, and V. splendidus were impaired in their ability to cause larval death compared to the wild type strains. Culture supernatants of Pseudomonas aeruginosa, known to contain several secreted proteases, showed virtually no toxicity to oyster larvae. Purified V. tubiashii protease A (VtpA), but not the prototype metalloprotease, thermolysin from Bacillus thermoproteolyticus, was highly toxic to the larvae. In addition, toxicity of purified VtpA was much greater for $6 \mathrm{~d}$ old oyster larvae than for $16 \mathrm{~d}$ old larvae. Together, these results indicated that culture supernatants of a variety of Vibrio species are highly toxic to oyster larvae and that the production of a metalloprotease is required for this effect. We propose that there are, as yet uncharacterized, specific substrates contained in larval tissue that are degraded by VtpA as well as certain homologous metalloproteases produced by other marine Vibrio species which, in turn, may contribute to vibriosis.
\end{abstract}

KEY WORDS: Vibrio tubiashii $\cdot$ VtpA $\cdot$ Vibriosis $\cdot$ Extracellular products $\cdot$ Shellfish hatchery

\section{INTRODUCTION}

Bacterial diseases can be a major cause of mortality in rearing facilities of molluscan bivalve larvae, with such outbreaks resulting in substantial economic loss for shellfish growers (Brown 1981, Elston et al. 1981, 2008, Elston 1999). In addition, losses of hatchery- and nursery-reared shellfish larvae and juveniles have, in some cases, been accompanied by reports of failures of natural shellfish larval populations (Elston 1984, Perkins 1993, Prado et al. 2005). Although bacterial strains from other genera, such as Pseudomonas, have been demonstrated to be pathogens of larval bivalves (Brown 1973), members of the genus Vibrio are the most frequent cause of larval mortalities. Overall, vibriosis is considered to be the most serious disease of hatchery-reared oyster larvae (Tubiash et al. 1970, Hada et al. 1984, Elston et al. 2008).
The genus Vibrio is composed of gram-negative curved rods that are motile by means of a single polar flagellum. The genus is widespread in the aquatic environment throughout the world and includes more than 30 species, many of which are pathogenic not only for humans but also for various aquatic animals, including fish and shellfish. Bacterial infections of adult mollusk bivalves only rarely lead to disease while larval and juvenile bivalves are highly susceptible to infection by some species of the genus Vibrio (Elston \& Leibovitz 1980, Nottage \& Birkbeck 1987, Elston et al. 2008).

Vibrio tubiashii recently re-emerged as a pathogen of several species of bivalve larvae, causing substantial economic loss, especially in Pacific oyster-rearing facilities (Estes et al. 2004, Elston et al. 2008). Vibriosis was initially termed bacillary necrosis (Tubiash et al. 1965) and the causative bacterium later designated as V. tubiashii by Hada et al. (1984). Vibriosis is characterized by a rapid onset of reduced larval motility, detached vela, 
and necrotic soft tissue, accompanied by high mortality rates, often exceeding $90 \%$ (Sindermann 1988). Although first identified as a cause of such mortalities in 1965 (Tubiash et al. 1965), V. tubiashii re-emerged in 2006 and 2007 as a causative agent for mass larval mortalities of Pacific oysters and other species in some hatcheries in the Pacific Northwest (Elston et al. 2008).

Extracellular metalloproteases produced by a variety of marine Vibrio species have been established as virulence factors for their fish or shellfish hosts. For instance, metalloprotease Vvp from a strain of $V$. vulnificus pathogenic for eels is essential for colonization of mucosal surfaces in aquatic animals (Valiente et al. 2008). In $V$. anguillarum, a metalloprotease has been shown to contribute to its ability to invade host fish tissues and cause systemic disease in a number of fish species (Norqvist et al. 1990). In addition, an extracellular metalloprotease of $V$. splendidus is toxic when injected into adult oysters (Le Roux et al. 2007, Binesse et al. 2008). Moreover, it has been reported that extracellular products produced by $V$. aestuarianus are responsible for its virulence for Crassostrea gigas (Labreuche et al. 2006b). A subsequent study (Labreuche et al. 2006a) showed that protease concentration from $V$. aestuarianus injected into oysters is correlated with a decrease in phagocytic activities in the host. Another study reported that toxicity of culture supernatants of $V$. proteolyticus to larvae of European flat oysters Ostrea edulis was due to an extracellular metalloprotease and that the enzyme also rapidly broke down gill tissue of blue mussels Mytilus edulis (Nottage \& Birkbeck 1987).

In a previous study, we reported that culture supernatants of Vibrio tubiashii were highly toxic to Pacific oyster larvae (Hasegawa et al. 2008). This toxicity was attributed to the activity of the secreted metalloprotease because (1) metalloprotease inhibitors, including EDTA, severely impaired the toxicity of $V$. tubiashii culture supernatants to Pacific oyster larvae, (2) culture supernatants of non-proteolytic $V$. cholerae carrying the $V$. tubiashii metalloprotease gene ( $v t p A)$ were highly toxic to Pacific oyster larvae, and (3) a VtpA ${ }^{-}$ mutant of $V$. tubiashii showed much reduced larval mortality. Thus, we concluded that the VtpA is a major virulence factor for Pacific oyster larvae.

In this study, we purified the VtpA protein and analyzed its enzymatic activity and toxicity to oyster larvae in more detail. We also examined larval toxicity of culture supernatants of various Vibrio species and their correlation with protease production, using both inhibitors and their relevant mutant strains. In addition, we analyzed non-Vibrio related metalloproteases, including culture supernatants of Pseudomonas aeruginosa and purified thermolysin from Bacillus thermoproteolyticus.

\section{MATERIALS AND METHODS}

Bacterial strains and growth conditions. Bacterial strains and plasmids are listed in Table 1. Vibrio tubiashii, $V$. alginolyticus, $V$. fluvialis, $V$. mimicus, $V$. splendidus, $V$. vulnificus, and $V$. parahaemolyticus were grown in Luria-Bertani (LB) media supplemented with $3 \%$ sodium chloride, whereas $V$. cholerae and Pseudomonas aeruginosa were grown in pure LB media. All Vibrio strains were grown at $30^{\circ} \mathrm{C}$ and $P$. aeruginosa was grown at $37^{\circ} \mathrm{C}$ overnight. Escherichia coli Top 10 cells (Invitrogen) were used for routine cloning as well as His-tagged protein purification and grown in LB broth or on agar supplemented with appropriate antibiotics. When required, antibiotics were supplemented as follows: ampicillin (Ap), $100 \mu \mathrm{g} \mathrm{ml}{ }^{-1}$; kanamycin $(\mathrm{Km}), 50 \mu \mathrm{g} \mathrm{ml}^{-1}$; chloramphenicol $(\mathrm{Cm}), 20 \mu \mathrm{g} \mathrm{ml}^{-1}$ and tetracycline (Tc), $10 \mu \mathrm{gl}^{-1}$.

Construction of the Vibrio tubiashii VtpA ${ }^{-}$mutant. To obtain a cosmid library of $V$. tubiashii, genomic DNA of $V$. tubiashii strain RE22 was partially digested with Sau3AI. DNA fragments (approximately $20 \mathrm{~kb}$ ) were ligated into pLAFR5 cosmid arms, which were produced by digesting pLAFR 5 with $S c a I$ and BamHI to produce 2 DNA fragments (Keen et al. 1988). The ligation mix was packaged into lambda phageheads in vitro (Packagene Lambda DNA Packaging System, Promega) and transduced into Escherichia coli strain LE392, selecting for TC-resistant colonies. The cosmid DNA containing the vtpA gene was obtained from the library by screening pools of colonies by standard PCR using vtpA-specific primers. A vtpA containing cosmid, pLAFR5-vtpA, was then mutagenized via the lambda red recombinase system (Datsenko \& Wanner 2000), using hybrid lambda red vtpA primers and pKD3 (Datsenko \& Wanner 2000) as the template. Competent cells of E. coli BW25113 carrying pKD46 (Datsenko \& Wanner 2000) and pLAFR5-vtpA were electroporated with a linear PCR product containing the $\mathrm{Cm}$ resistance cassette flanked by vtpA sequences. The resulting $\mathrm{Cm}$ and $\mathrm{Tc}$ resistance clones were confirmed for the correct insertion by PCR, creating pLAFR5-vtpA ${ }^{-}$. E. coli strain Top10 carrying pLAFR5vtpA $^{-}$was then conjugated into $V$. tubiashii RE22 by triparental matings with the helper $E$. coli strain HB101 carrying pRK2013 (Figurski \& Helinski 1979). $V$. tubiashii carrying the mutagenized cosmid were selected for the marker $(\mathrm{Cm})$ inserted into the vtpA gene and then tested for the loss of vector marker (Tc). The allelic exchange following double homologous recombination was confirmed by PCR. All PCR and cloning reactions were conducted using standard procedures (Ausubel et al. 1991, Saltikov \& Newman 2003). 
Purification of VtpA-His protein. A DNA fragment containing the entire coding region of $v t p A$ was PCR amplified from Vibrio tubiashii RE22 chromosomal DNA by using primers 5'-GAG GAA TAA TAA ATG AAA CAA CGT CAA ATG CTT TG-3' and 5'-GTC TAA TCT TAG TGT CAC GC-3', which do not encode the native stop codon. The fragment was then cloned into pBAD-His vector (Invitrogen), thus fusing a $6 \times$ His tag to the open reading frame (ORF) to yield pBADvtpA-His. The insert was sequenced to verify the construct. To purify VtpA-His from bacterial cells, Escherichia coli strain Top10 carrying pBADvtpA-His was grown in LB medium supplemented with arabinose $\left(0.2 \%\right.$, wt $\left.\mathrm{vol}^{-1}\right)$ and ampicillin at $37^{\circ} \mathrm{C}$ and induced for several $\mathrm{h}$. Cells from $250 \mathrm{ml}$ culture were typically harvested by centrifugation when the culture reached an absorbance of light $\left(A_{600}\right)$ value of 1.8 . VtpA-His protein from the lysed cell pellet was purified using Ni-NTA column (Qiagen), according to the manufacturer's instructions. Protein concentrations were determined by using Bradford Assay kit (Pierce). Thermolysin was purchased from Calbiochem.

Larvae toxicity assay. Stationary-phase bacterial cultures were centrifuged and the filter-sterilized supernatants, as well as purified proteins, were used as the samples. We aliquoted 6, 10, and $16 \mathrm{~d}$ old oyster larvae (Coast Seafoods Company) in sterile seawater to a 96-well flat-bottom plate (Nunc) at a density of approximately 20 larvae in $100 \mu \mathrm{l}$ per well. Toxicity to larvae was assessed by adding various amounts of undiluted or diluted supernatants or purified proteins $(0.5$ to $2 \mu \mathrm{l})$ to the wells in triplicate and varying incubation times (every 30 min up to $4 \mathrm{~h}$ ) at room temperature to determine the threshold for toxicity. The same amount of growth medium or protein elution buffer was used as a control. Toxicity to larvae was determined by visualization on an inverted microscope. We considered oyster larvae dead when the larvae stop moving, the velum was grossly damaged, and the larvae appeared to be darkened, similar to phenotypes described in Garland et al. (1983). The concentration of EDTA used in these experiments was $10 \mathrm{mM}$.

Enzyme assays. Vibrio species supernatants and purified proteins were assayed for proteolytic activities using $1 \%$ azocasein as previously described by Hasegawa et al. (2008). The proteolytic units were calculated as the ratio between the absorbances from azocasein assays (at $420 \mathrm{~nm}$ ) and the normalized bacterial growth $(600 \mathrm{~nm})$. For semiquantative assays for gelatinase, elastase, and mucinase activities, wells were

Table 1. Vibrio spp., Pseudomonas spp., Escherichia spp. Bacterial strains and plasmids used in this study. Ap ${ }^{\mathrm{r}}$ : ampicillin resistant; $\mathrm{Tc}^{\mathrm{r}}$ : tetracycline resistant; $\mathrm{Cm}^{\mathrm{r}}$ : chloramphenicol resistant; $\mathrm{Mob}^{+}$: can be mobilized; $\mathrm{Tra}^{+}$: capable of self transfer; $\mathrm{Km}^{\mathrm{r}}$ : Kanamycin resistant

\begin{tabular}{|c|c|c|}
\hline Strains/Plasmids & Relevant characteristics & Source/ Reference \\
\hline \multicolumn{3}{|l|}{ Strains } \\
\hline V. tubiashii RE22 & Wild-type & Estes et al. (2004) \\
\hline V. tubiashii VtpA17 & $\mathrm{VtpA}^{-}$derivative of RE22 & Present study \\
\hline V. cholerae C7258 & Wild-type & Robert et al. (1996) \\
\hline V. cholerae 638 & HapA $^{-}$derivative of $\mathrm{C} 7258$ & Robert et al. (1996) \\
\hline V. splendidus LGP32 & Wild-type & Le Roux et al. (2007) \\
\hline V. splendidus $\delta 2989$ & Vsm$^{-}$derivative of LGP32 & Le Roux et al. (2007) \\
\hline V. alginolyticus ATCC17749 & Wild-type & Sakazaki (1968) \\
\hline$V$. fluvialis $1959-82$ & Wild-type & Hickman-Brenner et al. (1984) \\
\hline V. mimicus ATCC33653 & Wild-type & Davis et al. (1981) \\
\hline V. parahaemolyticus ATCC17802 & Wild-type & Fujino et al. (1974) \\
\hline V. vulnifucus ATCC27562 & Wild-type & Amaro et al. (1992) \\
\hline$P$. aeruginosa PA14 & Wild-type & Rahme et al. (1995) \\
\hline P. aeruginosa $\Delta \mathrm{LasB}$ & LasB $^{-}$derivative of PA14 & M. Schuster (pers. comm.) \\
\hline P. aeruginosa $\Delta \mathrm{LasR}$ & LasR $^{-}$derivative of PA14 & M. Schuster (pers. comm.) \\
\hline E. coli Top10 & Cloning host & Invitrogen \\
\hline E. coli BW25113 & Conjugation donor & Datsenko \& Wanner (2000) \\
\hline \multicolumn{3}{|l|}{ Plasmids } \\
\hline pBAD-His & $A p^{r}$, protein expression vector & Invitrogen \\
\hline pBADvtpA-His & $\mathrm{Ap}^{\mathrm{r}}, \quad$ tpA gene in pBAD-His & Present study \\
\hline pLARF5 & $\mathrm{Tc}^{\mathrm{r}}$, cosmid cloning vector & Keen et al. (1988) \\
\hline pLAFR5-vtpA & $\mathrm{Tc}^{\mathrm{r}}, v t p A$ gene of RE22 in pLARF5 & Present study \\
\hline pLAFR5- $\Delta$ vtpA & $\mathrm{TC}^{\mathrm{r}}, \mathrm{Cm}^{\mathrm{r}}, v \operatorname{tp} A^{-}$derivative of pLAFR5-vtpA & Present study \\
\hline pKD3 & $\begin{array}{l}\mathrm{Cm}^{\mathrm{r}} \text {, template for PCR amplification for red } \\
\text { recombinase-mediated recombination }\end{array}$ & Datsenko \& Wanner (2000) \\
\hline pKD46 & $\mathrm{Ap}^{\mathrm{r}}$, plasmid encoding red recombinase & Datsenko \& Wanner (2000) \\
\hline pRK2013 & $\mathrm{Mob}^{+} \mathrm{Tra}^{+} \mathrm{Km}^{\mathrm{r}}$ & Figurski \& Helinski (1979) \\
\hline
\end{tabular}


made in $1 \%$ substrate-containing agarose media and the bottoms of the wells were sealed with agarose. Supernatant samples were applied to the wells and the plates were incubated at $30^{\circ} \mathrm{C}$. After $24 \mathrm{~h}$, halos around the wells due to protease activity became visible in the assay plates. A reaction was scored as positive with at least $1 \mathrm{~mm}$ of visible halo. For the collagenase assay, $100 \mu \mathrm{l}$ of supernatant was incubated with $400 \mu \mathrm{l}$ of $1 \%$ Hide-Remazol Brilliant Blue R (Sigma) for $30 \mathrm{~min}$ at $37^{\circ} \mathrm{C}$. The reactions were centrifuged and the absorbances at $595 \mathrm{~nm}$ were measured. When added, the concentration of EDTA was $10 \mathrm{mM}$. Proteolytic activity was also assessed using Zymogram gel electrophoresis as described previously (Hasegawa et al. 2008).

\section{RESULTS}

\section{Toxicity of culture supernatants}

Previously, we documented that culture supernatants of Vibrio tubiashii are highly toxic to Pacific oyster larvae due to the production of VtpA (Hasegawa et al. 2008). In addition, sequence analyses revealed that VtpA homologs are widespread amongst other Vibrio species (Hasegawa et al. 2008). To determine if culture supernatants of other Vibrio species show toxicity to oyster larvae that can be attributed to metalloprotease activities, we tested sterile culture supernatants from various marine Vibrio species in azocasein as well as larval toxicity assays. Also included in this study was a wellstudied metalloprotease, elastase from Pseudomonas aeruginosa, known to be enzymatically and structurally related to this group of Vibrio metalloproteases (Häse \& Finkelstein 1990, Miyoshi \& Shinoda 2000, Cahan et al. 2001). As expected, the azocasein assay showed that culture supernatants of $V$. fluvialis, $V$. mimicus, $V$. vulnificus, $V$. tubiashii, V. cholerae, V. splendidus, and $P$. aerugionosa all contained considerable levels of extracellular proteases (Fig. 1A). When assayed for toxicity to $10 \mathrm{~d}$ old oyster larvae, all protease-containing culture supernatants of the tested Vibrio species caused substantial levels of mortality (Fig. 1B). Appropriate media were used as the negative control in this assay, which showed no toxicity to oyster larvae (data not shown). There was no dramatic difference between $V$. tubiashii and these Vibrio

A

$\mathrm{B}$ species in their proteolytic activities or their resulting toxicities. On the contrary, culture supernatants of $V$. alginolyticus and $V$. parahaemolyticus under our growth conditions did not contain significant azocasein activities and did not show considerable toxicity to oyster larvae (Fig. 1).

It has been known that elastase, a metalloprotease produced by Pseudomonas aeruginosa, is similar to Vibrio metalloproteases (Miyoshi \& Shinoda 2000), showing $64 \%$ similarity to the VtpA (data not shown), which is much lower than similarities between Vibrio metalloproteases (81 to $87 \%$, Hasegawa et al. 2008). Although P. aeruginosa produced high levels of azocasein activity, only minimal larvae mortality (ca. 13\%) was observed (Fig. 1B); however, an unusual effect was observed, resulting in loss of overall larval movement, which the cilia were still actively moving (data not shown). A mutation in lasR (quorum sensing regulator), but not lasB (elastase), eliminated this effect
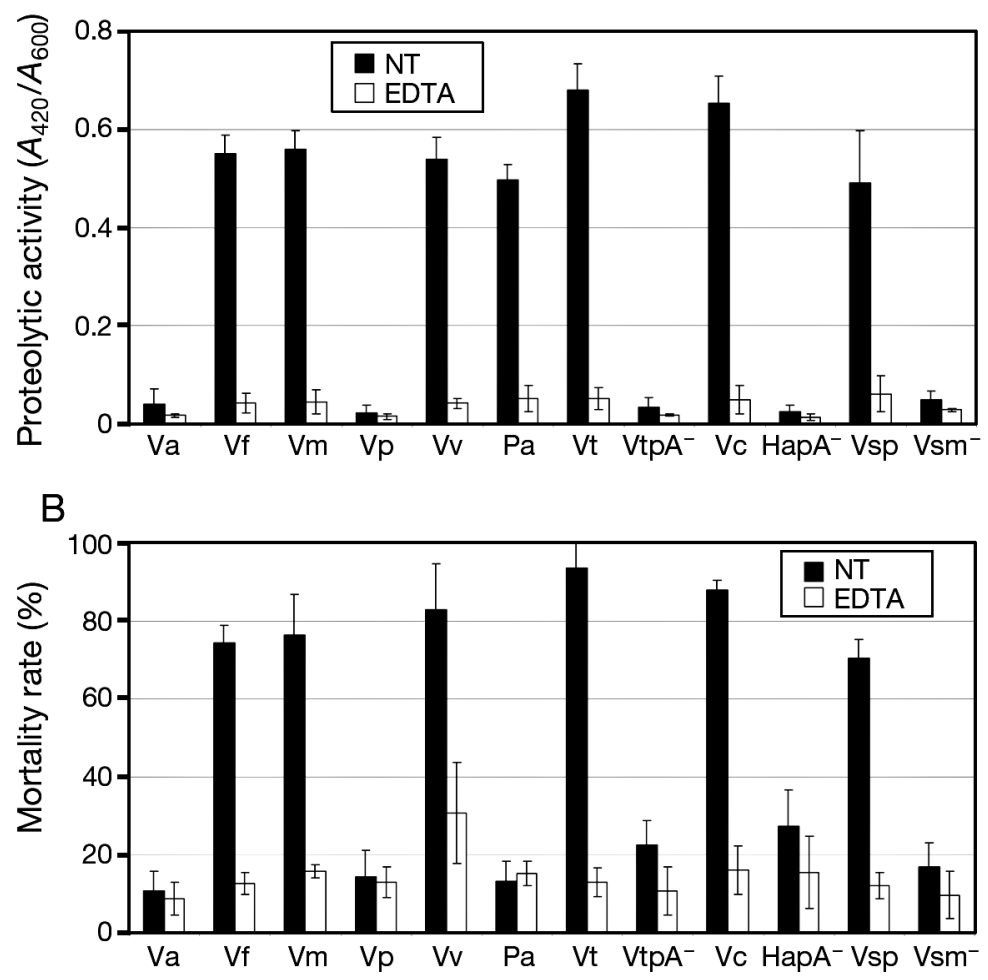

Fig. 1. Vibrio spp. and Pseudomonas aeruginosa. Analyses of supernatants. (A) Protease activity and (B) toxicity were assayed in absence or presence of $10 \mathrm{mM}$ EDTA. All cells were harvested at late stationary phase. Proteolytic and toxic activities were determined using azocasein and $10 \mathrm{~d}$ old oyster larvae, respectively, as described in 'Materials and methods.' Va: V. alginolyticus; Vf: V. fluvialis;: Vm: V. mimicus; $\mathrm{Vp}: V$. parahaemolyticus; Vv: V. vulnificus; Pa: P. aeruginosa; Vt: V. tubiashii; $\mathrm{VtpA}^{-}$: the $\mathrm{VtpA}^{-}$mutant of $\mathrm{Vt}_{\text {; }} \mathrm{Vc}$ : $V$. cholerae; HapA ${ }^{-}$: the HapA ${ }^{-}$mutant of $\mathrm{Vc}_{i}$ Vsp: V. splendidus; $\mathrm{Vsm}^{-}$: the $\mathrm{Vsm}^{-}$mutant of Vsp. $A_{420}, A_{600}$ : absorbance of light at 420 and $600 \mathrm{~nm}$ respectively. NT: non-treated. For the toxicity assay, filter-sterilized supernatants were added to final concentrations of $1 \%$. Error bars indicate SD $(\mathrm{N}=3)$ 
(data not shown). Our previous study demonstrated that the metalloprotease inhibitor, EDTA, strongly reduced the proteolytic activity and thus the toxicity level of culture supernatants of V.tubiashii (Hasegawa et al. 2008). Treatment with EDTA not only significantly reduced the proteolytic activities of all tested supernatants (Fig. 1A), but also severely diminished toxicity levels of all the Vibrio species tested (Fig. 1B). By contrast, EDTA treatment did not affect the unusual toxicity observed for $P$. aeruginosa supernatants (data not shown).

\section{Substrate specificity of culture supernatants}

In order to correlate culture supernatant toxicity with digestion of various potentially physiologically relevant substrates, culture supernatants from various marine Vibrio species and Pseudomonas aeruginosa were examined for activity toward gelatin, elastin, mucin, casein, and collagen. Except for casein, all of the potential substrates are thought to be found in oysters (Galtsoff 1964). Table 2 shows the various substrate specificities of the tested supernatants. For Vibrio species tested, supernatants exhibiting larval toxicity also exhibited gelatinase, caseinase, and collagenase, but not elastase or mucinase, activities. Moreover, the $P$. aeruginosa supernatants that showed very limited toxicity to oyster larvae also degraded gelatin, elastin, casein, and collagen.

\section{Effects of loss of the metalloprotease on pathogenicity}

To further validate the role of metalloproteases in the observed toxicity of Vibrio supernatants, we analyzed isogenic $V$. cholerae and $V$. splendidus strains carrying mutations in their metalloprotease genes (hapA and vsm, respectively) with high homology to VtpA. In addition, we generated a novel, stable mutant of $V$. tubiashii by deleting the entire vtpA gene. Proteolytic activity of this mutant strain was fully restored by providing the vtpA gene in trans (data not shown). Fig. 1A shows that compared to the wild type, these metalloprotease deficient mutants produced only minimal levels of extracellular protease, which were comparable to those of the parent strains treated with EDTA. Zymographic analysis using gelatin as the substrate showed that proteolytic band profiles varied among these species (Fig. 2). In $V$. cholerae and $V$. splendidus, virtually all proteolytic bands disap- peared in their mutant samples, whereas the $V$. tubiashii mutant still retained some observable proteolytic bands (Fig. 2). Similar to our previous results (Hasegawa et al. 2008), the V. tubiashii mutant showed dramatically reduced toxicity to oyster larvae (Fig. 1B). Likewise, the $V$. cholerae and $V$. splendidus mutants showed much-reduced larval toxicity compared to the parent strains (Fig. 1B).

\section{Proteolytic activity and toxicity of purified His-tagged VtpA}

Although we had evidence that VtpA was directly responsible for larval mortality observed in culture supernatants of Vibrio tubiashii (Hasegawa et al. 2008), we wanted to independently confirm the toxicity of this enzyme. To determine if VtpA is a structural

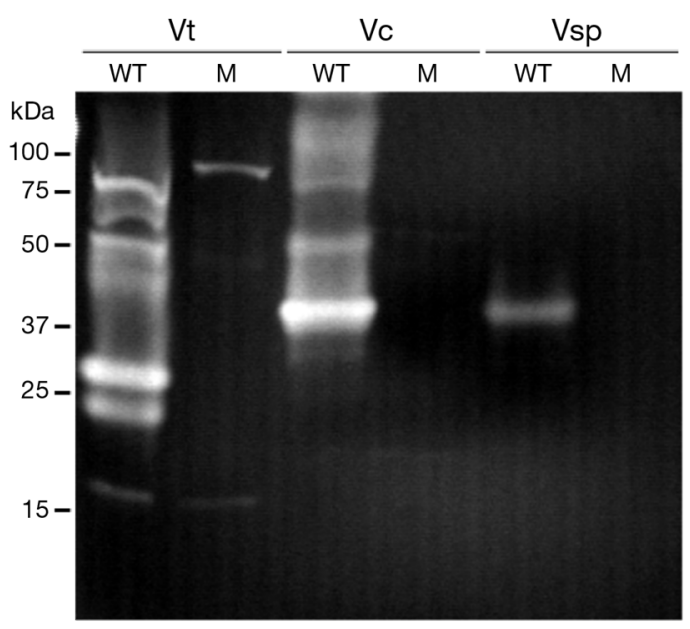

Fig. 2. Vibrio spp. and Pseudomonas aeruginosa. Zymography analysis of supernatants using a $10 \%$ gelatin polyacrylamide gel. WT: wild type strain, M: the metalloprotease mutant. Bands of proteolytic activity in the zymogram gel are shown as clear protein bands in a dark background. The molecular masses $(\mathrm{kDa})$ on the left indicate the positions of molecular weight markers 
toxin for oyster larvae, a His-tagged VtpA was purified and tested for toxicity. To test toxicity of another similar metalloprotease ( $41 \%$ similarity to the VtpA, data not shown) produced by a gram-positive bacterial species, we used thermolysin, a prototype metalloprotease from Bacillus thermoproteolyticus. Fig. 3A shows that both the thermolysin and purified VtpA presented high azocasein activities. On the other hand, VtpA was highly toxic to oyster larvae, whereas thermolysin was found to be much less toxic to oyster larvae in spite of high azocasein activities (Fig. 3B).

\section{Relationship between proteolytic activities and toxicity levels in the purified VtpA}

Clearly, certain metalloproteases from various marine Vibrio species are toxic to oyster larvae. To determine the relative potency of these metalloproteases, serial dilutions of protease containing samples, including culture supernatants and purified protease preparations, were tested for their toxicity. Azocasein units required to produce 50 or $20 \%$ of mortality in Pacific oyster larvae are summarized in Table 3 . The levels of proteolytic activities required to kill $50 \%$ of the larvae were very similar for the $V$. tubiashii and $V$. cholerae supernatants, as well as the VtpA purified protein. By contrast, thermolysin required an approximately 10-fold higher concentration of protease units than VtpA to achieve $50 \%$ larval mortality. Similarly, V. tubiashii, V. cholerae, and VtpA produced $20 \%$ mortality at virtually equivalent protease units and were approximately 16 -fold more lethal than thermolysin.

\section{Effects of larval ages on susceptibility to VtpA}

Several reports indicated that larval and juvenile stages of oysters are more susceptible to vibriosis than the adult stages (Nottage \& Birkbeck 1987, Elston \& Leibovitz 1980, Elston et al. 2008, Gómez-León et al. 2008). To examine if VtpA toxicity varies amongst different ages of oyster larvae, mortality rates of $6 \mathrm{~d}$ old and $16 \mathrm{~d}$ old larvae were analyzed using various dilutions of VtpA. At higher VtpA concentrations, no major difference was observed in mortality rate of both age groups, with over $90 \%$ of larvae being killed in either age group (Table 4). Even at the highest concentration used, a certain number of larvae appeared to be unaffected (data not shown), suggesting natural resistance or absence of the, as yet unidentified, VtpA substrates in these oysters. At lower concentrations of VtpA (50 and $25 \mathrm{ng} \mathrm{ml}^{-1}$ ), a significant difference in survival rates was observed between the different ages. Overall, 6 d old oyster larvae were much more susceptible to the effects of VtpA compared to $16 \mathrm{~d}$ old oyster larvae (Table 4). Moreover, sensitivity of $24 \mathrm{~h}$ old larvae was comparable to that of $6 \mathrm{~d}$ old larvae (data not shown).

\section{DISCUSSION}

Bacterial diseases are a major cause of mortality of bivalve mollusk larvae and may result in major losses and great expense for shellfish growers. Members of the genus Vibrio are the most frequent cause of these mortalities. In 2006 and 2007, some of the commercial oyster hatcheries on the west coast of the United States
A

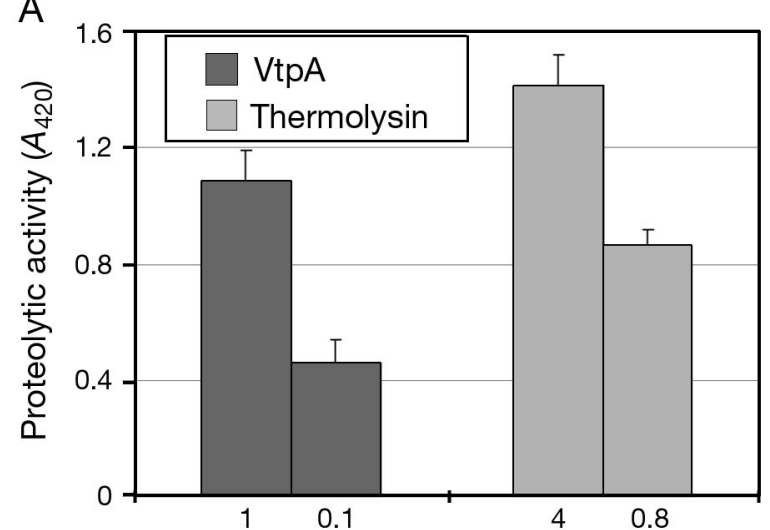

B

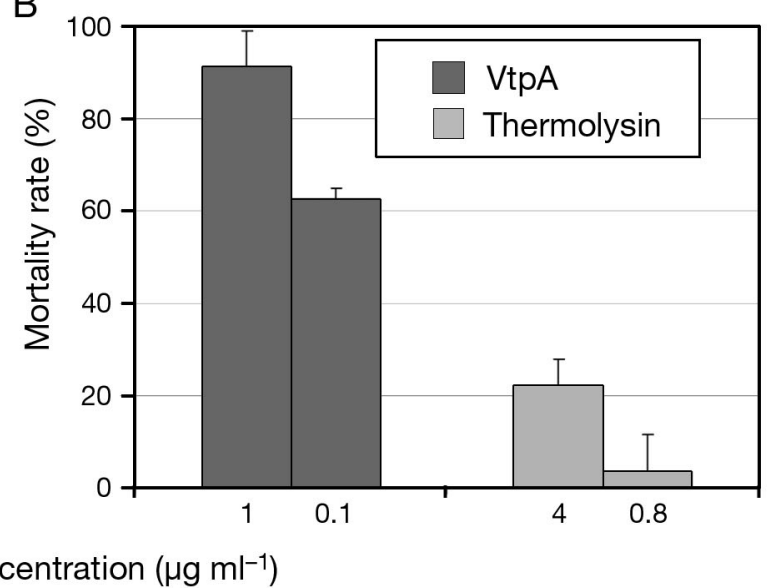

Fig. 3. Vibrio spp. and Crassostrea gigas. Analyses of purified V. tubiashii protease A (VtpA) and thermolysin. Quantitative assays for (A) proteolytic activities and (B) oyster toxicity of VtpA, and thermolysin at 2 different concentrations. Proteolytic and toxic activities were determined using azocasein and $10 \mathrm{~d}$ old oyster larvae, respectively, as described in 'Materials and methods.' $A_{420}$ : light absorbance at $420 \mathrm{~nm}$. Error bars indicate $\mathrm{SD}(\mathrm{N}=3)$ 
Table 3. Vibrio spp. and Crassostrea gigas. Proteolytic units (absorbance at $420 \mathrm{~nm}, A_{420}$ ) to kill 50 and $20 \%$ of Pacific oyster larvae. Data are means \pm SD based on at least 3 independent experiments. sup: supernatants

\begin{tabular}{|lrr|}
\hline \multirow{2}{*}{$\begin{array}{l}\text { Culture sup } \\
\text { or purified proteins }\end{array}$} & \multicolumn{2}{c|}{ Azocasein unit $\left(A_{420}\right)$} \\
& & \\
& & \\
V. tubiashii sup & $0.351 \pm 0.08$ & $0.129 \pm 0.06$ \\
$V$. cholerae sup & $0.364 \pm 0.05$ & $0.148 \pm 0.08$ \\
VtpA & $0.368 \pm 0.01$ & $0.152 \pm 0.09$ \\
Thermolysin & $3.28 \pm 0.10$ & $2.146 \pm 0.32$ \\
\hline
\end{tabular}

were affected by outbreaks of $V$. tubiashii caused vibriosis, and resulted in a markedly reduced seed supply of Pacific oysters resulted (Elston et al. 2008). In spite of the economic importance of Vibrio species in the cultivation of larval and juvenile bivalves in shellfish hatcheries, very little is known about the virulence mechanisms employed by these pathogens, nor their presence at shellfish hatcheries. We have recently shown that the metalloprotease, but not the cytolysin, acts as one of the critical factors for the toxicity of $V$. tubiashii supernatants on Pacific oyster larvae (Hasegawa et al. 2008). However, we could not rule out the possibility that VtpA actually interacts with other still unknown factors, which, in turn, might cause toxicity to the larvae. Therefore, it was essential to prove that the VtpA is a structural toxin. A recent study shows that a purified metalloprotease from V. splendidus is toxic when injected into abductor muscles of adult oysters (Binesse et al. 2008). Here, we have also reported, for the first time, that simple exposure to metalloproteases from a variety of marine Vibrio species causes larval mortality. Previous studies have shown that the metalloproteases are potentially lethal to adult oysters when injected (Le Roux et al. 2007, Binesse et al. 2008). Moreover, we extended our previous observations by analyzing proteolytic profiles and larval toxicity of culture supernatants from other marine Vibrio species as well as similar metalloproteases from Bacillus and Pseudomonas.

Our data indicated that culture supernatants of multiple Vibrio species were highly toxic to Pacific oyster larvae, which was attributed to the production of metalloproteases. Our previous analyses showed not only that a non-pathogenic bacterial isolate lacked the ability to produce high levels of extracellular protease, but that a number of marine Vibrio species, including $V$. cholerae, V. fluvialis, V. splendidus, and V. vulnificus, possess VtpA homologs in their genome (Hasegawa et al. 2008). Culture supernatants of these species were all toxic to oyster larvae and their effects were strongly reduced in the presence of EDTA, indicating that secreted metalloproteases are involved in these observed toxicities to oyster larvae. On the other hand, culture supernatants of $V$. alginolyticus and $V$. parahaemolyticus did not produce high levels of azocasein activities and no toxicity, further supporting the concept that metalloproteases are mostly responsible for larval toxicity. However, these results do not exclude the possibility that other strains of these species are toxic to larvae. Interestingly, culture supernatants of a metalloprotease (elastase) producer, Pseudomonas aeruginosa, caused only minimal larval mortality, although they stopped the overall movement of the larvae. Culture supernatants of an elastase (LasB) deficient mutant of $P$. aeruginosa also caused this effect on the larvae, whereas a quorum sensing (LasR) deficient mutant did not. LasR is a transcriptional regulator which controls a number of virulence factors (Juhas et al. 2005), indicating that this effect of $P$. aeruginosa supernatants on the larvae is not due to the metalloprotease elastase, but to other factors controlled by quorum sensing in $P$. aeruginosa, such as pyocyanin. Together, these results indicated that culture supernatants of many Vibrio species exhibited potentially high toxic effects to oyster larvae, though those of $P$. aeruginosa did not.

Our previous findings revealed that culture supernatants of a VtpA mutant of Vibrio tubiashii showed much reduced toxicity to oyster larvae (Hasegawa et al. 2008). However, since the previous VtpA minus strain was an insertional mutant, a new stable deletion mutant was created in this study. In addition, isogenic mutants carrying deletions in metalloprotease genes of $V$. cholerae and V. splendidus were analyzed. Culture supernatants of not only the VtpA mutant but also $V$. cholerae and $V$. splendidus metalloprotease mutants showed a marked loss of toxicity compared to their respective wild type strains, further implying that the metalloproteases from other Vibrio species are toxic to oyster larvae. Our previous sequence analyses revealed that these metalloproteases share high levels of similarity, in particular, throughout the mature pro-

Table 4. Vibrio spp. and Crassostrea gigas. Mortality of different ages of oyster larvae induced by $V$. tubiashii protease A (VtpA). $A_{420}$ : absorbance at $420 \mathrm{~nm}$. Data are means $\pm \mathrm{SD}$ based on at least 3 independent experiments

\begin{tabular}{|lccc|}
\hline $\begin{array}{l}\text { VtpA } \\
\text { concentration } \\
\left(\text { ng ml }^{-1}\right)\end{array}$ & $\begin{array}{c}\text { Calculated } \\
\text { proteolytic } \\
\text { units }\left(A_{420}\right)\end{array}$ & $\begin{array}{c}\text { Mortality (\%) of VtpA to } \\
6 \text { d old } \\
\text { oyster }\end{array}$ & $\begin{array}{c}\text { 16 d old } \\
\text { oyster }\end{array}$ \\
\hline 500 & 0.543 & $96.3 \pm 4.4$ & $91.6 \pm 8.3$ \\
250 & 0.272 & $95.9 \pm 5.3$ & $88.1 \pm 4.7$ \\
125 & 0.136 & $90.2 \pm 14.2$ & $78.3 \pm 6.2$ \\
50 & 0.054 & $86.5 \pm 10.3$ & $50.8 \pm 9.1^{\mathrm{a}}$ \\
25 & 0.027 & $71.2 \pm 8.9$ & $28.5 \pm 12.5^{\mathrm{a}}$ \\
a Data for larval mortalities were evaluated by Student's \\
$t$-test (p < 0.01 compared with the 6 d old larvae)
\end{tabular}


tease region (Hasegawa et al. 2008), suggesting that these metalloproteases target a similar substrate that results in killing the oyster larvae. It appears that the elastase of Pseudomonas aeruginosa does not utilize the putative target protein(s). Amongst the tested supernatants, differences in substrate specificity were noted that did not match the observed toxicity. Consistent with previous observation (Binesse et al. 2008), cytotoxicity assays using Vero cells showed strong toxic effects of $V$. tubiashii supernatants as well as a purified VtpA protein (authors' unpubl. data). Future study will focus on identifying the oyster proteins targeted by these toxic metalloproteases.

In this study, we have conclusively demonstrated that the VtpA metalloprotease is a structural toxin against oyster larvae. However, it is interesting to note that the prototype metalloprotease, thermolysin, did not efficiently kill larvae, again suggesting differences in substrate specificity for these different metalloproteases. Importantly, these results also conclusively show that the VtpA alone can kill oyster larvae. The importance of proteases, in particular metalloproteases, in the pathogenicity of fish and shellfish diseases caused by marine bacteria has been widely reported (Nottage \& Birkbeck 1987, Norqvist et al. 1990, Thompson et al. 2005, Labreuche et al. 2006a, Le Roux et al. 2007, Binesse et al. 2008, Hasegawa et al. 2008, Valiente et al. 2008).

Clearly, bacterial proteases produced by various marine Vibrio species can play important roles in shellfish disease. However, it is not clear how specific these enzymes are for their respective targets found on different shellfish. To compare the relative toxicity of the $V$. tubiashii protease to those produced by other Vibrio species, the doses of protease required to kill 50 and $20 \%$ of oyster larvae, respectively, were determined for $V$. tubiashii and $V$. cholerae supernatants. In addition, purified VtpA protein and thermolysin were compared. Overall, no major difference was observed for the 2 Vibrio species proteases, whereas thermolysin required a much higher dose to produce equivalent larval mortality. We conclude that the majority of the toxicity observed in culture supernatants of $V$. tubiashii can be attributed to the direct activity of VtpA on the larvae. Moreover, it appears that, at least for $V$. cholerae, other metalloproteases are just as efficient at killing larvae as the $V$. tubiashii metalloprotease.

It has been reported that sensitivity of different stages of shellfish development to vibriosis varies, with larval and juvenile stages being more susceptible than adult oysters (Elston \& Leibovitz 1980, Gómez-León et al. 2008). Consistent with these reports, our results showed that resistance to VtpA significantly increased with oyster age. Our studies further revealed that the previously known age-dependent sensitivity to vibrio- sis may in fact be due to the activities of the metalloprotease. Moreover, these differences indicate that the putative targets degraded by the metalloprotease are modified or differentially expressed in oysters as they develop with ages. Additionally, differences in larval sensitivity might be due to changes in the accessibility to the protease targets, or due to active production by oyster larvae of molecules counteracting or inhibiting protease activity. For future study, we plan to utilize our purified VtpA to determine the exact nature of the oyster proteins affected by the metalloprotease.

In this report, we conclusively showed, for the first time, that the Vibrio tubiashii metalloprotease itself is directly toxic to oyster larvae. We also observed that a variety of Vibrio species produce related metalloproteases that can potentially kill oyster larvae. These highly similar proteases appeared to be equally efficient at inducing larval toxicity. However, not all metalloproteases are toxic, with the Pseudomonas aeruginosa elastase and Bacillus thermoproteolyticus thermolysin being virtually non-toxic. Age of the oyster larvae affected their susceptibility to the toxic metalloprotease, with older larvae being more resistant. Further studies will be required to fully understand the underlying mechanisms of oyster toxicity produced by various metalloproteases.

Acknowledgements. We thank Dr. R. A. Elston for providing the Vibrio tubiashii strain as well as many helpful insights. We thank Dr. F. Le Roux, Dr. M. Schuster, Dr. A. J. Silva, Dr. Y.-C. $\mathrm{Su}$, and Dr. B. Wanner for generously providing strains used in this study. We are also grateful to Coast Seafoods Company, Quilcene, WA, for generously providing Pacific oyster larvae. This study was partially funded by grants from the Oregon Sea Grant, USDA/CREES Animal Health and Diseases, and the Agricultural Research Foundation.

\section{LITERATURE CITED}

Amaro C, Biosca EG, Esteve C, Fouz B, Toranzo AE (1992) Comparative study of phenotypic and virulence properties in Vibrio vulnificus biotype 1 and 2 obtained from a European eel farm experiencing mortalities. Dis Aquat Org 13: 29-35

Ausubel FM, Brent R, Kingston RE, Moore DD, Seidman JG, Smith JA, Truhl KS (1991) Current protocols in molecular biology. John Wiley and Sons, New York

Binesse J, Delsert C, Saulnier D, Champomier-Vergès MC and others (2008) The metalloprotease Vsm is the main toxic factor for Vibrio splendidus extracellular products. Appl Environ Microbiol 74:7108-7117

Brown C (1973) The effects of some selected bacteria on embryos and larvae of the American oyster, Crassostrea virginica. J Invertebr Pathol 21:215-223

Brown C (1981) A study of two shellfish-pathogenic Vibrio strains isolated from a Long Island hatchery during a recent outbreak of disease. J Shellfish Res 1:83-87

- Cahan R, Axelrad I, Safrin M, Ohman DE, Kessler E (2001) A secreted aminopeptidase of Pseudomonas aeruginosa. 
Identification, primary structure, and relationship to other aminopeptidases. J Biol Chem 276:43645-43652

Datsenko KA, Wanner BL (2000) One-step inactivation of chromosomal genes in Escherichia coli K-12 using PCR products. Proc Natl Acad Sci. USA 97:6640-6645

Davis BR, Fanning RG, Madden JM, Steigerwalt AG, Bradford HB, Smith HL, Brenner DJ (1981). Characterization of biochemically atypical Vibrio cholerae strains and designation of a new pathogenic species, Vibrio mimicus. J Clin Microbiol 14:631-639

Elston RA (1984) Prevention and management of infectious diseases in intensive mollusc husbandry. J World Mariculture Soc 15:284-300

Elston RA (1999) Development, histology and health management of seed oysters. World Aquaculture Society, Baton Rouge, LA

Elston RA, Leibovitz L (1980) Pathogenesis of experimental vibriosis in larval American oysters, Crassostrea gigas. Can J Fish Aquat Sci 37:964-978

Elston RA, Leibovitz L, Relyea D, Zatila J (1981) Diagnosis of vibriosis in a commercial oyster hatchery epizootic, a case history. Aquaculture 24:53-62

Elston RA, Hasegawa H, Humphrey KL, Polyak IK, Häse CC (2008) Re-emergence of Vibrio tubiashii in bivalve shellfish aquaculture: severity, environmental drivers, geographic extent and management. Dis Aquat Org 82: 119-134

Estes RM, Friedman CS, Elston RA, Herwig RP (2004) Pathogenicity testing of shellfish hatchery bacterial isolates on Pacific oyster Crassostrea gigas larvae. Dis Aquat Org 58:223-230

Figurski DH, Helinski DR (1979) Replication of an origincontaining derivative of plasmid RK2 dependent on a plasmid function provided in trans. Proc Natl Acad Sci USA 76:1648-1652

Fujino T, Sakazaki R, Tamura K (1974) Designation of the type strain of Vibrio parahaemolyticus and description of 200 strains of the species. Int J Syst Bacteriol 24:447-449

Galtsoff PS (1964) Organs of disgestion and food of the oyster. Fish Bull 64:219-235

Garland CD, Nash GV, Summer CE, McMeekin TA (1983) Bacterial pathogens of oyster larvae (Crassostrea gigas) in a Tasmanian hatchery. Aust J Mar Freshw Res 34:483-487

Gómez-León J, Villamil L, Salger SA, Sallum RH, RemachaTriviño A, Leavitt DF, Gómez-Chiarri M (2008) Survival of eastern oysters Crassostrea virginica from three lines following experimental challenge with bacterial pathogens. Dis Aquat Org 79:95-105

Hada HS, West PA, Lee JV, Stemmler J, Colwell RR (1984) Vibrio tubiashii sp. nov., a pathogen of bivalve mollusks. Int J Syst Bacteriol 34:1-4

Häse CC, Finkelstein RA (1990) Comparison of the Vibrio cholerae hemagglutinin/protease and the Pseudomonas aeruginosa elastase. Infect Immun 58:4011-4015

Hasegawa H, Lind EJ, Boin MA, Häse CC (2008) The extracellular metalloprotease of Vibrio tubiashii is a major virulence factor for Pacific oyster (Crassostrea gigas) larvae. Appl Environ Microbiol 74:4101-4110

Hickman-Brenner FW, Brenner DJ, Steigerwalt, AG, Schreiber $M$ and others (1984) Vibrio fluvialis and Vibrio furnissii isolated from a stool sample of one patient. J Clin Microbiol 20:125-127

Juhas M, Eberl L, Tümmler B (2005) Quorum sensing: the power of cooperation in the world of Pseudomonas. Environ Microbiol 7:459-471
Keen NT, Tamaki S, Kobayashi D, Trollinger D (1988) Improved broad-host-range plasmids for DNA cloning in Gram-negative bacteria. Gene 70:191-197

Labreuche Y, Lambert C, Soudant P, Boulo V, Huvet A, Nicolas, JL (2006a) Cellular and molecular hemocyte responses of the Pacific oyster, Crassostrea gigas, following bacterial infection with Vibrio aestuarianus strain 01/32. Microbes Infect 8:2715-2724

> Labreuche Y, Soudant P, Gonçalves M, Lambert C, Nicolas JL (2006b) Effects of extracellular products from the pathogenic Vibrio aestuarianus strain 01/32 on lethality and cellular immune responses of the oyster Crassostrea gigas. Dev Comp Immunol 30:367-379

> Le Roux F, Binesse J, Saulnier D, Mazel D (2007) Construction of a Vibrio splendidus mutant lacking the metalloprotease gene vsm by use of a novel counterselectable suicide vector. Appl Environ Microbiol 73:777-784

> Miyoshi S, Shinoda S (2000) Microbial metalloproteases and pathogenesis. Microbes Infect 2:91-98

$>$ Norqvist A, Norrman B, Wolf-Watz H (1990) Identification and characterization of a zinc metalloprotease associated with invasion by the fish pathogen Vibrio anguillarum. Infect Immun 58:3731-3736

Nottage AS, Birkbeck TH (1987) The role of toxins in Vibrio infections of bivalve mollusca. Aquaculture 67:244-246

Perkins FO (1993) Infectious diseases of mollusks. In: Couch JA, Fournie JW (eds) Pathobiology of marine and estuarine organisms. CRC Press, Boca Raton, FL, p 255-287

> Prado S, Romalde JL, Montes J, Barja JL (2005) Pathogenic bacteria isolated from disease outbreaks in shellfish hatcheries. First description of Vibrio neptunius as an oyster pathogen. Dis Aquat Org 67:209-215

Rahme LG, Stevens EJ, Wolfort SF, Shao J, Tompkins RG, Ausubel FM (1995) Common virulence factors for bacterial pathogenicity in plants and animals. Science 268: 1899-1902

Robert A, Silva A, Benitez JA, Rodriguez BL and others (1996) Tagging a Vibrio cholerae El Tor candidate vaccine strain by disruption of its hemagglutinin/protease gene using a novel reporter enzyme: Clostridium thermocellum endoglucanase A. Vaccine 14:1517-1522

Sakazaki R (1968) Proposal of Vibrio alginolyticus for biotype 2 Vibrio parahaemolyticus. Jpn J Med Sci Biol 21: $359-362$

> Saltikov CW, Newman DK (2003) Genetic identification of a respiratory arsenate reductase. Proc Natl Acad Sci USA 100:10983-10988

Sindermann CJ (1988) Vibriosis of larval oysters. In: Sindermann CJ, Lightner DV (eds) Disease diagnosis and control in North American aquaculture. Elsevier, Amsterdam, p 271-274

Thompson FL, Klose KE, the AVIB Group (2005) Vibrio2005: the First International Conference on the Biology of Vibrios. J Bacteriol 188:4592-4596

Tubiash HS, Chanley PE, Leifson E (1965) Bacillary necrosis, a disease of larval and juvenile bivalve mollusks. I. Etiology and epizootiology. J Bacteriol 90:1036-1044

Tubiash HS, Colwell RR, Sakazaki R (1970) Marine vibrios associated with bacillary necrosis, a disease of larval and juvenile bivalve mollusks. J Bacteriol 103:2721-2723

> Valiente E, Lee CT, Hor LI, Fouz B, Amaro C (2008) Role of the metalloprotease Vvp and the virulence plasmid pR99 of Vibrio vulnificus serovar E in surface colonization and fish virulence. Environ Microbiol 10:328-338

Submitted: December 17, 2008; Accepted: April 7, 2009

Proofs received from author(s): May 27, 2009 